\title{
Magnetically driven anisotropic structural changes in the atomic laminate Mn2GaC
}

\author{
Martin Dahlqvist, Arni Sigurdur Ingason, Björn Alling, F. Magnus, Andreas Thore, Andrejs \\ Petruhins, Aurelija Mockuté, U. B. Arnalds, M. Sahlberg, B. Hjorvarsson, Igor Abrikosov \\ and Johanna Rosén
}

\section{Linköping University Post Print}

N.B.: When citing this work, cite the original article.

Original Publication:

Martin Dahlqvist, Arni Sigurdur Ingason, Björn Alling, F. Magnus, Andreas Thore, Andrejs Petruhins, Aurelija Mockuté, U. B. Arnalds, M. Sahlberg, B. Hjorvarsson, Igor Abrikosov and Johanna Rosén, Magnetically driven anisotropic structural changes in the atomic laminate Mn2GaC, 2016, Physical Review B. Condensed Matter and Materials Physics, (93), 1, 014410. http://dx.doi.org/10.1103/PhysRevB.93.014410

Copyright: American Physical Society

http://www.aps.org/

Postprint available at: Linköping University Electronic Press http://urn.kb.se/resolve?urn=urn:nbn:se:liu:diva-124463 


\title{
Magnetically driven anisotropic structural changes in the atomic laminate $\mathrm{Mn}_{2} \mathrm{GaC}$
}

\author{
M. Dahlqvist, ${ }^{1,}$ A. S. Ingason,,${ }^{1, *}$ B. Alling, ${ }^{1}$ F. Magnus, ${ }^{2}$ A. Thore,${ }^{1}$ A. Petruhins,,${ }^{1}$ A. Mockute,${ }^{1}$ U. B. Arnalds,${ }^{3}$ \\ M. Sahlberg, ${ }^{4}$ B. Hjörvarsson, ${ }^{2}$ I. A. Abrikosov, ${ }^{5,6,7}$ and J. Rosen ${ }^{1}$ \\ ${ }^{1}$ Thin Film Physics, Department of Physics, Chemistry and Biology, Linköping University, SE-581 83, Linköping, Sweden \\ ${ }^{2}$ Department of Physics and Astronomy, Uppsala University, Box 530, SE-751 21, Uppsala, Sweden \\ ${ }^{3}$ Science Institute, University of Iceland, Dunhaga 3, IS-107, Reykjavik, Iceland \\ ${ }^{4}$ Department of Chemistry, The Ångström Laboratory, Uppsala University, Box 538, SE-751 21, Uppsala, Sweden \\ ${ }^{5}$ Theoretical Physics, Department of Physics, Chemistry and Biology, Linköping University, SE-581 83, Linköping, Sweden \\ ${ }^{6}$ Materials Modeling and Development Laboratory, National University of Science and Technology “MISIS,” 119049, Moscow, Russia \\ ${ }^{7}$ LOCOMAS Laboratory, Tomsk State University, 634050, Tomsk, Russia
}

(Received 23 October 2015; published 8 January 2016)

\begin{abstract}
Inherently layered magnetic materials, such as magnetic $M_{n+1} A X_{n}$ (MAX) phases, offer an intriguing perspective for use in spintronics applications and as ideal model systems for fundamental studies of complex magnetic phenomena. The MAX phase composition $M_{n+1} A X_{n}$ consists of $M_{n+1} X_{n}$ blocks separated by atomically thin $A$-layers where $M$ is a transition metal, $A$ an A-group element, $X$ refers to carbon and/or nitrogen, and $n$ is typically 1,2 , or 3 . Here, we show that the recently discovered magnetic $\mathrm{Mn}_{2} \mathrm{GaC}$ MAX phase displays structural changes linked to the magnetic anisotropy, and a rich magnetic phase diagram which can be manipulated through temperature and magnetic field. Using first-principles calculations and Monte Carlo simulations, an essentially one-dimensional (1D) interlayer plethora of two-dimensioanl (2D) Mn-C-Mn trilayers with robust intralayer ferromagnetic spin coupling was revealed. The complex transitions between them were observed to induce magnetically driven anisotropic structural changes. The magnetic behavior as well as structural changes dependent on the temperature and applied magnetic field are explained by the large number of low energy, i.e., close to degenerate, collinear and noncollinear spin configurations that become accessible to the system with a change in volume. These results indicate that the magnetic state can be directly controlled by an applied pressure or through the introduction of stress and show promise for the use of $\mathrm{Mn}_{2} \mathrm{GaC}$ MAX phases in future magnetoelectric and magnetocaloric applications.
\end{abstract}

DOI: 10.1103/PhysRevB.93.014410

\section{INTRODUCTION}

Since the work of Ising [1], magnetism in low-dimensional systems has been the subject of extensive theoretical [2-5] and experimental [6-9] research. As an example, monolayers of $3 d$ transition metals on hexagonal single crystal substrates are good candidates for the physical realization of frustrated two dimensional (2D) itinerant antiferromagnetic (AFM) systems [5,9-11]. This frustration, or the inability for the material to satisfy the competing magnetic exchange interactions (MEI) simultaneously, can result in spin-glass behavior, noncollinear magnetic order, and more complex spin textures such as spin spirals and skyrmions [12-18]. Such spin textures can be manipulated with very small current densities and have therefore been suggested for magnetic storage and logic technologies $[13,19,20]$.

The ability to manipulate the magnetic interactions is key to obtaining control over the complex magnetic behavior observed in such systems [21,22]. Materials containing Mn are of interest in this respect as they have been found to exhibit sharp magnetic and structural transitions, and allow the utilization of such transitions in refrigeration [23]. The magnetocaloric effect [24], i.e., the change in temperature of a material with a magnetic field, has been exploited and is characteristic

\footnotetext{
* Correspondence and requests for materials should be addressed to M.D. (madah@ifm.liu.se) or A.S.I (arnsi@ifm.liu.se)
}

of ferromagnets such as $\mathrm{Mn}_{3} A C$ ( $\left.A=\mathrm{Al}, \mathrm{Zn} \mathrm{Ga}, \mathrm{Ge}, \mathrm{Sn}, \mathrm{In}\right)$ [25,26], $\mathrm{MnFe}(\mathrm{As}, \mathrm{P})$ [27], and $\mathrm{Mn}(\mathrm{As}, \mathrm{Sb})$ [28]. In MnP, it was found that a particular Mn-Mn separation plays the dominant role in determining the change from AFM to ferromagnetic (FM) order [29], in turn suggesting an approach for tuning metamagnetism in similar systems [30,31]. Layered systems are also important as interactions through alternating magnetic and nonmagnetic (NM) materials can be tuned via layer roughness [32], thickness [33], and elemental composition [34].

Other Mn containing materials, i.e., $R \mathrm{MnO}_{3}(R=\mathrm{Y}$, Dy, $\mathrm{Tb}, \mathrm{Ho}, \mathrm{Er}, \mathrm{Tm}, \mathrm{Yb}$, and Lu) [35,36], exhibit a magnetoelectric effect, with both ferroelectric and magnetic order. Extensive work has been conducted to find such materials, including man-made composite layer systems, which make use of magnetostriction to convert the magnetic action to an electric response [20], which is important for the advancement of the state-of-art spin-electronic technologies [16].

Recently, a new magnetic material, $\mathrm{Mn}_{2} \mathrm{GaC}$, was theoretically predicted and subsequently synthesized as a heteroepitaxial thin film [37]. This material has a hexagonal structure of $\mathrm{Mn}-\mathrm{C}-\mathrm{Mn}$ trilayers interleaved with an atomic layer of $\mathrm{Ga}$ atoms, which results in a Mn-C-Mn-Ga-Mn-C-Mn-Ga atomic-layer stacking in the $c$ direction. $\mathrm{Mn}_{2} \mathrm{GaC}$ belongs to a group of materials known as MAX phases where $M$ is a transition metal, $A$ an A-group element, and $X$ refers to carbon and/or nitrogen. Generally MAX phases combine ceramic and metallic properties [38] and are routinely synthesized both 
as bulk [39] and as thin films [40]. $\mathrm{Mn}_{2} \mathrm{GaC}$ can as such be combined with other (non) magnetic MAX phases in a variety of heterostructures, thus making it an ideal model system for the study of complex magnetic phenomena that occur in atomically layered materials.

In this work we study the magnetic and structural behavior of $\mathrm{Mn}_{2} \mathrm{GaC}$ at and below room temperature (RT) using vibrating sample magnetometry (VSM), magnetooptical Kerr effect (MOKE), and x-ray diffraction (XRD). In addition, a magnetic ground-state search was conducted using firstprinciples density functional theory (DFT) and Heisenberg Monte Carlo simulations to provide a better understanding of magnetism in these atomically laminated MAX phase materials [41-50]. We find magnetically driven anisotropic structural changes and strong indications of complex magnetic behavior, which can be manipulated through temperature and magnetic field.

\section{METHODS}

\section{A. Magnetic measurements}

The magnetic response of the samples was measured in a Cryogenic Ltd. vibrating sample magnetometer (VSM) in the temperature range 3 to $300 \mathrm{~K}$. Magnetization was recorded in a magnetic field up to $5 \mathrm{~T}$, with the field applied both parallel and perpendicular to the film plane. The magnetic response of the sample holder and bare $\mathrm{MgO}$ substrate was measured independently. Below $10 \mathrm{~K}$ the linear diamagnetic response of the substrate changes drastically to paramagnetic at low fields. This background was fitted with an arctangent function with a linear component. The substrate response was measured at $2 \mathrm{~K}$ intervals from the lowest temperature up to $15 \mathrm{~K}$ and fitted to this model and then this was used to subtract from the measured signal of the sample to obtain the signal from the film. Magnetooptic Kerr effect (MOKE) measurements were carried out in the longitudinal geometry with $s$ polarized light. Full hysteresis loops up to the maximum available field of $400 \mathrm{mT}$ were recorded over the temperature range 5 to $380 \mathrm{~K}$, and the remanent magnetization extracted from the hysteresis loops. The sample was rotated around the azimuthal angle to examine the presence of in-plane magnetic anisotropy. Thin film samples within this work were heteroepitaxially grown on $\mathrm{MgO}(111)$ at $550^{\circ} \mathrm{C}$ by magnetron sputtering using three confocal sources with elemental targets. Further synthesis details are given in in Ref. [37].

\section{B. X-ray measurements}

$\mathrm{X}$-ray diffraction (XRD) measurements were performed on a Bruker D8 diffractometer equipped with a Våntec position sensitive detector (PSD) with $4^{\circ}$ opening using $\mathrm{Cu} \mathrm{K}_{\alpha 1}$ radiation. Measurements were performed in temperatures from 50 to $300 \mathrm{~K}$ using an Oxford Phenix cryostat and in a $2 \theta$ range of 20 to $50^{\circ}$.

\section{First-principles calculations}

For the stability calculations presented several spin configurations were considered including NM, FM, and multiple AFM configurations; single layer AFM with spins changing sign for every $M$-atom layer, corresponding to AFM[0001 $]_{1}$, multilayered AFM ordering of $\alpha$ consecutive $M$ layers (where $\alpha=2,4,6,8)$ with the same spin direction before changing sign upon crossing an $A$ or an $X$ layer (AFM[0001] $]_{\alpha}^{\mathrm{A}}$ and $\left.\mathrm{AFM}[0001]_{\alpha}^{\mathrm{X}}\right)$, and antiparallel spins within one $M$ layer (in-AFM1 and in-AFM2). PM states are often considered as NM, although local magnetic moments are often preserved above the transition temperature in an uncorrelated fashion. In this work the PM state of $\mathrm{Mn}_{2} \mathrm{GaC}$ was modelled by using the disorder local moment (DLM) [51,52] method by having spin-correlation functions equal, or at least close, to zero on the first eight $M$-coordination shells. The disorder in magnetic moments in $\left(\mathrm{Mn}_{0.5}^{\uparrow} \mathrm{Mn}_{0.5}^{\downarrow}\right)_{2} \mathrm{GaC}$ is simulated by means of the special quasirandom structure method using a supercell with $64 \mathrm{Mn}, 32 \mathrm{Ga}$, and $32 \mathrm{C}$ atoms, i.e., $4 \times 4 \times 1$ or $16 M_{2} A X$ unit cells [52-54]. The schematic illustration and spin correlation functions defining the collinear spin configurations are shown in Fig. 1 and Table S1 of the Supplemental Materials, respectively [55]. Details of the considered noncollinear spin configurations are found in Table S2 [55].

All first-principles calculations reported here are carried out using the projector augmented wave (PAW) [56] method as implemented within the Vienna ab initio simulation package (VASP) [57,58]. We adopted both the spin-polarized generalized gradient approximation (GGA), as parameterized by Perdew-Burke-Ernzerhof (PBE), and the local spin density approximation (LSDA or in this work just LDA) for treating electron exchange and correlation effects [59]. Wave functions are expanded in a plane-wave basis set with an energy cutoff of $400 \mathrm{eV}$. For sampling of the Brillouin zone we used the Monkhorst-Pack scheme [60] on a grid of $23 \times 23 \times 7[1 \times 1$ $\times 1$ unit cell (uc)], $23 \times 23 \times 5(1 \times 1 \times 2 \mathrm{uc}), 23 \times 23 \times 3(1$ $\times 1 \times 3$ uc $), 23 \times 23 \times 3(1 \times 1 \times 4$ uc $), 11 \times 23 \times 7(2 \times 1 \times$ $1 \mathrm{uc})$, and $5 \times 5 \times 5(4 \times 4 \times 1 \mathrm{uc}) k$ points. The convergence thresholds were $10^{-5} \mathrm{eV}$ and $10^{-4} \mathrm{eV}$ per fu for electronic and ionic relaxations, respectively. We optimized the structure of $\mathrm{Mn}_{2} \mathrm{GaC}$ for each magnetic configuration by minimizing the total energy with respect to volume, $c / a$ ratio, and internal parameters. Possible effects from spin-orbit coupling were not considered, as these effects are believed to be in the sub-meV range for these types of materials [61].

\section{Heisenberg Monte Carlo simulations}

The coarse-grained model used for the magnetic groundstate search within the canonical ensemble is illustrated in Fig. S1 [55] where the local moment of Mn atoms in a Mn-C-Mn trilayer plane is represented by a supermoment. The identified spin configurations of lowest energy, FM and AFM[0001] $]_{\alpha}^{\mathrm{A}}$, all have parallel spin directions within their Mn-C-Mn trilayer, thus we only focus on the magnetic exchange interactions (MEI) across the $A$ layer along the $c$ axis. The spin correlation function $\Phi_{\alpha}$ for the five considered spin configurations are given in Table S3 [55]. The critical temperature cannot be modelled using our simplified approach since the MEI within a Mn-C-Mn tri-layer is neglected, and hence the exact temperature of the Monte Carlo simulation becomes irrelevant as it scales with the area of each layer. The focus is instead of finding possible magnetic ground-state configurations using a Heisenberg Hamiltonian $\mathcal{H}=-\sum(i \neq j) J_{i j} \mathbf{e}_{i} \cdot \mathbf{e}_{j}$, where 
(a)
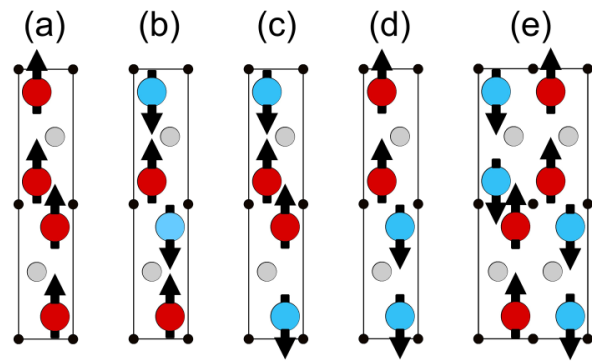

(g)

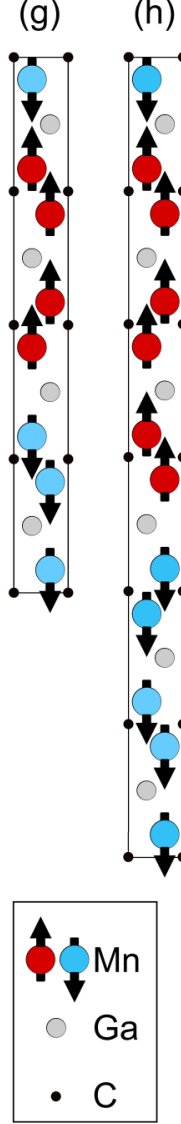

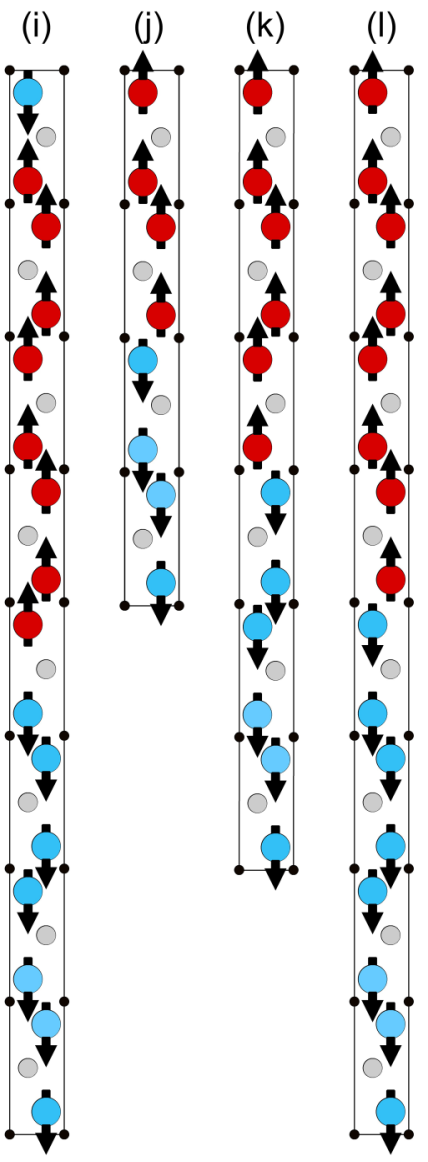

FIG. 1. Schematic illustration of considered collinear magnetic spin configurations for (a) FM, (b) AFM[0001 $]_{1}$, (c) AFM[0001 $]_{2}^{A}$, (d) AFM $[0001]_{2}^{X}$, (e) in-AFM1, (f) in-AFM2, (g) AFM[0001 $]_{4}^{A}$, (h) $\mathrm{AFM}[0001]_{6}^{A}$, (i) AFM $[0001]_{8}^{A}$, (j) AFM $[0001]_{4}^{X}$, (k) AFM $[0001]_{6}^{X}$, and (1) AFM $[0001]_{8}^{X}$ seen along the $[11 \overline{2} 0]$ direction. Spin directions of $\mathrm{Mn}$ atoms are indicated by up and down arrows.

$J_{i j}$ is the MEI between pairs of super-moments $(i, j)$, with unit vectors $\mathbf{e}_{i}$ and $\mathbf{e}_{j}$ along the local magnetic moment at site $i$ and $j$, represented by a chain of super-moments. We derived the exchange interactions $J_{\mathrm{ij}}$, i.e., MEI, between the supermoments of the Heisenberg Hamiltonian for the first four supermoment interlayer coordination shells at various volumes, by using the magnetic Connolly-Williams structure inversion method [62,63] in combination with energyvolume data. The volume dependence $J_{\mathrm{ij}}$ is displayed in Fig. S2 [55].

To allow for effects from long-range interaction on the magnetic structure, we chose to investigate chains consisting of 8 to 36 supermoments using periodic boundary conditions.
The temperature was initially set to a large value and slowly cooled towards $0 \mathrm{~K}$ to avoid possible metastable solutions. At each volume the chain lengths of minimum energy were selected for further analysis.

\section{RESULTS}

\section{A. Magnetic characterization using VSM}

Figure 2 shows the in-plane magnetization measured with VSM for temperatures (a) between 3 and $50 \mathrm{~K}$ and (b) from $50 \mathrm{~K}$ to $300 \mathrm{~K}$, of an approximately 100-nm thick $\mathrm{Mn}_{2} \mathrm{GaC}$ heteroepitaxial film grown on $\mathrm{MgO}$ (111) [37]. Note that previously published data in Ref. [37] only showed data for $\pm 150 \mathrm{mT}$. The $\mathrm{MgO}$ background contribution has been subtracted; for details see Fig. S3 [55]. In Fig. 2(a) at $3 \mathrm{~K}$ and at low fields we observe an FM response, shown clearly in the inset. This is followed by a gradual increase in magnetization with field that seems close to saturation at $5 \mathrm{~T}$ (the maximum available field). The rate of this increase is temperature dependent. The observed remanent magnetization (magnetization at zero field) $m_{r}$ corresponds to $\sim 0.3 \mu_{B}$ per $\mathrm{Mn}$ atom, which is low compared to the highest measured moment of $1.7 \mu_{B}$ per $\mathrm{Mn}$ at $3 \mathrm{~K}$ and $5 \mathrm{~T}\left(m_{5 \mathrm{~T}}\right)$, thus $m_{r}$ accounts for only $\sim 1 / 6^{\text {th }}$ of $m_{5 \mathrm{~T}}$. The measured moment of $1.7 \mu_{B}$ is comparable to, e.g., $2.12 \mu_{B}$ for Fe and $0.608 \mu_{B}$ for $\mathrm{Ni}$. The observed FM component is stable up to $230 \mathrm{~K}$, after which $m_{r}$ disappears. The inset in Fig. 2(b) shows $m_{5 \mathrm{~T}}$ as a function of temperature. A strong temperature dependence is seen in the 3 to $50 \mathrm{~K}$ regime, after which it is relatively constant around $0.3 \mu_{B}$ per Mn. Figure 2(b) shows that a drastic change in magnetic behavior takes place in the high temperature regime. Note that the scale is different in Figs. 2(a) and 2 (b) and the response at $50 \mathrm{~K}$ is shown in both. Above $230 \mathrm{~K}$ the material undergoes a magnetic field driven transition at high field, which is temperature dependent, arriving at a similar magnetization as $m_{r}$ at 3 to $200 \mathrm{~K}$. Given the high structural quality of the film [37], the effects of sample quality (point defects, grain boundaries, stacking faults, dislocations, etc.) are assumed not to influence the magnetic behavior.

The observed magnetic response cannot be described within a simple collinear framework. First to note is the low temperature response that cannot be regarded as traditional ferromagnetism. The small FM component, only accounting for $\sim 1 / 6^{\text {th }}$ of the total magnetization, and the gradual increase of the magnetization with field cannot be explained in terms of magnetic anisotropy. This would either require an easy axis (or cone) with a component perpendicular to the plane or strong in-plane anisotropy, most likely related to the crystal structure (magnetocrystalline anisotropy). Out-of-plane measurements, seen in Fig. S4 [55] for selected temperatures, show very low susceptibility at zero field, i.e., no remanence, as compared to what is observed in-plane. This rules out the first possibility. A second possibility would imply an easy/hard axis along one of the in-plane crystalline directions (with six-fold symmetry) giving a normalized remanent moment of at least $m_{r} / m_{5 \mathrm{~T}}=$ $\cos 30^{\circ}$ or 0.86 . This is noticeably larger than the observed $m_{r}$ of $\sim 1 / 6^{\text {th }}$ of the magnetization at high fields. Additionally, in-plane measurements with the sample rotated by $90^{\circ}$ around the azimuthal angle show no difference in behavior, thus ruling 

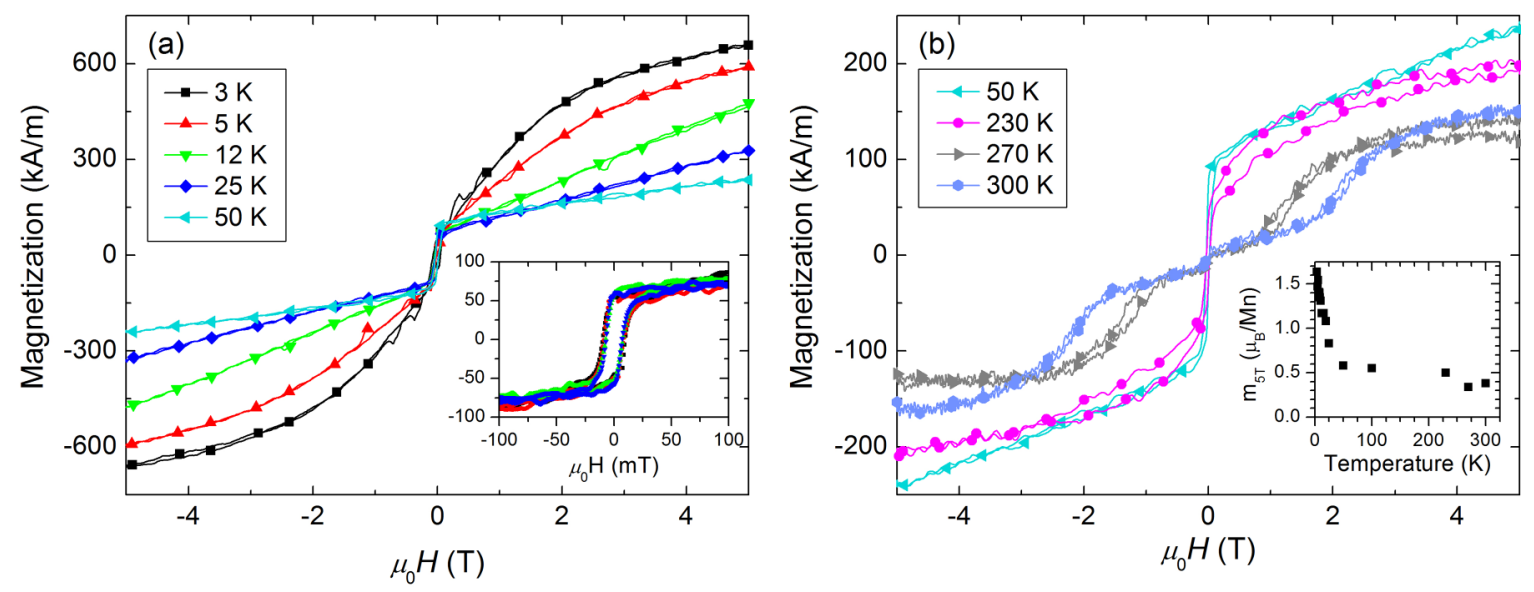

FIG. 2. The in-plane magnetization measured with VSM for selected temperatures in the range (a) 3 to $50 \mathrm{~K}$ and (b) 50 to $300 \mathrm{~K}$. Below $230 \mathrm{~K}$ a sharp FM transition is seen, see inset in (a) at low fields, followed by an increasing magnetization with field. Above $250 \mathrm{~K}$ there is no FM response and a new field-driven transition is observed. The inset in panel (b) shows the magnetic moment at $5 \mathrm{~T}, m_{5 \mathrm{~T}}$, as a function of temperature. Note that symbols used for the different temperatures in (a) and (b) are only shown for every 50 to 100 data points whereas the lines represent every data point.

out any strong in-plane magnetocrystalline anisotropy. The strong field-dependent behavior of the magnetization could be explained somewhat by the presence of large superparamagnetic clusters with different in-plane easy axis. However, the magnetic transition at $230 \mathrm{~K}$ cannot be characterized as a normal FM-PM (paramagnetic) transition as the magnetization curves above $230 \mathrm{~K}$ show that the system still has magnetic order and it is therefore unlikely that the system is PM below this temperature. For example, at $300 \mathrm{~K}$, the transitions at $\pm 2 \mathrm{~T}$ warrant further consideration as they show abrupt field-driven changes in the magnetic ordering. To explain this behavior further we therefore need additional information, and turn our attention to first-principles calculation.

\section{B. First-principles calculations}

Figure 3(a) shows the total energy as a function of volume for NM, FM, 11 different AFM, and PM spin configurations (see Fig. 1 and Table S1 [55] for the definition of spin configurations). Several ferrimagnetic configurations were considered. However, they all relax to either FM or to one of the considered AFM with equivalent Mn moments. The energies are given relative to the NM energy minimum $E_{0}^{\mathrm{NM}}$. The equilibrium total energy spans over a range of $100 \mathrm{meV}$ per atom, from NM to the lowest energy state $\mathrm{AFM}[0001]_{4}^{A}$. In-plane AFMs, AFM[0001] $]_{1}$, and PM (DLM) are found $\sim 50 \mathrm{meV} /$ atom below the NM state. Further down the $\mathrm{AFM}[0001]_{\alpha}^{X}$ are located, and the set of spin configurations of lowest energy correspond to FM and AFM[0001 $]_{\alpha}^{A}$. Importantly, we observe that all the identified low-energy collinear spin configurations have parallel spin directions within a Mn-C-Mn trilayer. This means that the intralayer FM coupling in $\mathrm{Mn}_{2} \mathrm{GaC}$ is robust. Moreover, the low-energy magnetic configurations are significantly more energetically stable than the DLM state, indicating that a complete disorder of magnetic moments has high energy cost, and that the transition temperature to the paramagnetic state should be relatively high, above the room temperature, in agreement with the experiment.
In Fig. 3(b) the crystal structure schematics are displayed for selected low-energy spin configurations, $\mathrm{FM}, \mathrm{AFM}[0001]_{4}^{A}$, and $\mathrm{AFM}[0001]_{2}^{A}$. Also shown are three different interlayer distances where $d_{X}$ is the Mn-Mn distance across a $C(X)$ layer, and $d_{A}^{+/+}$and $d_{A}^{+/-}$the Mn-Mn distances across a $\mathrm{Ga}(A)$ layer with $\mathrm{Mn}$ spins either parallel $(+/+)$ or antiparallel $(+/-)$. In Fig. 3(c) these interlayer distances, $d_{A}^{+/+}$and $d_{A}^{+/-}$, are plotted as a function of volume for FM, AFM $[0001]_{4}^{A}$, and AFM $[0001]_{2}^{A}$. Crucially, we find that $d_{X}$ is constant $(\sim 2.09 \AA)$ for all three spin configurations within this volume range. AFM[0001 $]_{4}^{A}$ displays a difference in $d_{A}^{+/+}$ and $d_{A}^{+/-}$, which implies that the spin alignment across the $A$ layers influences the $\mathrm{Mn}-\mathrm{Ga}-\mathrm{Mn}$ interlayer distance. As a result, the AFM $[0001]_{4}^{A}$ magnetic configuration can no longer be described with the original structure as the two Mn-Ga-Mn spacings are different and the magnetic unit cell becomes the defining structural entity with a different symmetry.

For the nine spin configurations of lowest energy in Fig. 3(a), FM, AFM[0001 $]_{\alpha}^{A}$, and AFM[0001 $]_{\alpha}^{X}$, the relaxed lattice parameters $c$ and $a$ are shown as a function of energy difference relative to $E_{0}^{\mathrm{NM}}$ in Fig. 3(d). All configurations have the same in-plane lattice parameter $a, \sim 2.9 \AA$, in agreement with the measured value [37], but different out-of-plane parameters $c$. This would mean that any stress in the film remains constant during transitions between magnetic states since coinciding structural changes only occur out of the plane. Furthermore, the spin configurations of lowest energy are close to degenerate, indicating a magnetic frustration in the system that could be influenced with field or temperature. Since different magnetic configurations have different lattice parameters and symmetries, the magnetic transitions should coincide with structural changes that could be probed with XRD.

\section{Structural and magnetic changes with temperature}

Figure 4(a) shows a $2 \theta-\omega$ XRD scan measured at RT and at $150 \mathrm{~K}$. At RT the peaks represent the $\mathrm{Mn}_{2} \mathrm{GaC}$ and the 

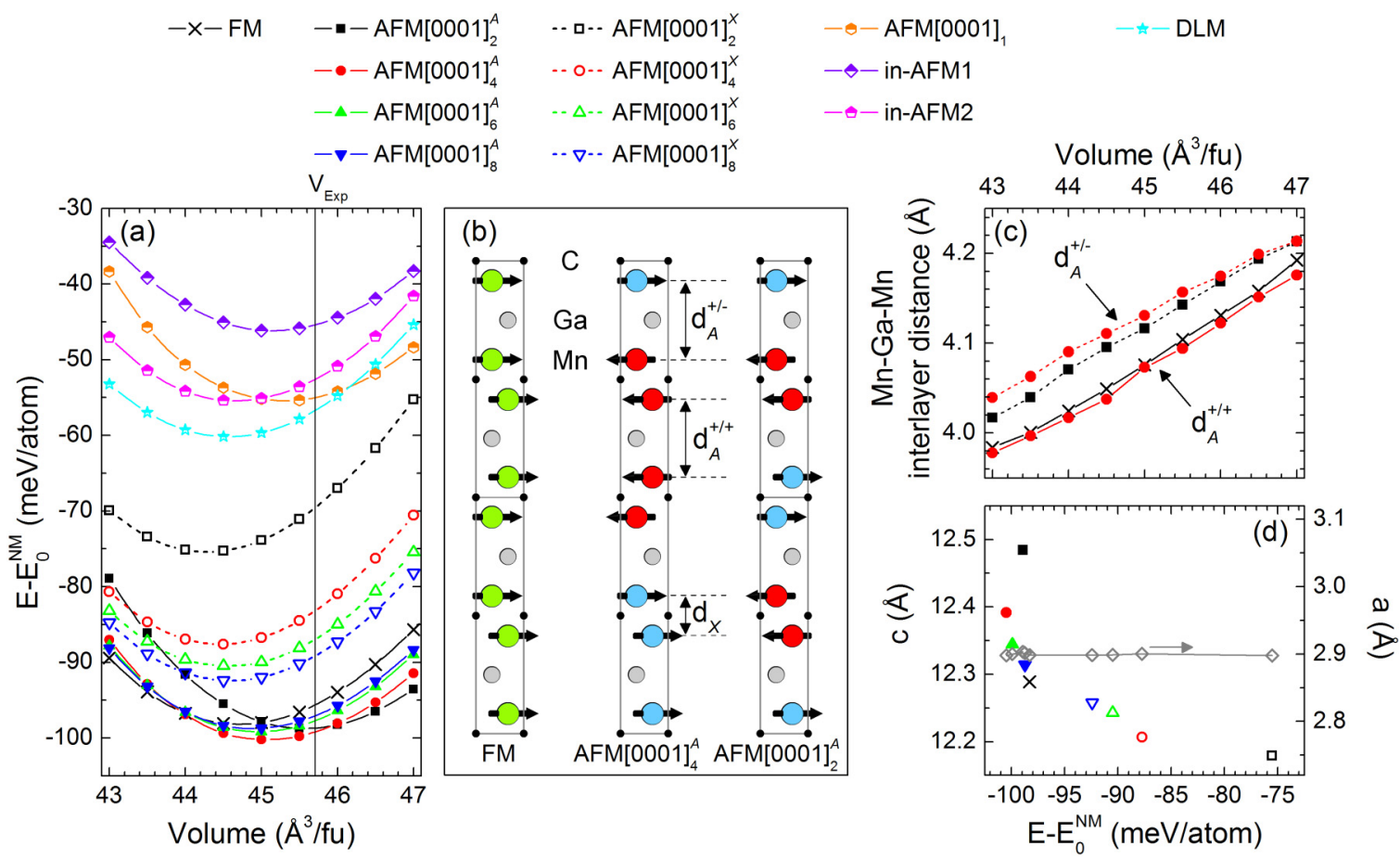

FIG. 3. Stability and structure from first-principles calculations. (a) Energy as a function of volume for different collinear spin configurations of $\mathrm{Mn}_{2} \mathrm{GaC}$. Energies are given relative to the minimum energy of the nonmagnetic state $E_{0}^{\mathrm{NM}}$. The measured volume at room temperature is shown by a vertical line [37]. (b) Magnetic unit cell schematics of selected low-energy spin configurations showing three different interlayer distances within the lattice. In (c) the Mn-Ga-Mn interlayer distance is displayed as a function of volume for the three configurations displayed in (b) showing two distinct distances, $d_{A}^{+/+}$and $d_{A}^{+/-}$, for the AFM[0001 $]_{4}^{A}$. Panel (d) shows the $c$ and $a$ lattice parameters as a function of energy difference for relaxed FM, AFM $[0001]_{\alpha}^{A}$, and $\mathrm{AFM}[0001]_{\alpha}^{X}$ spin configurations. All data presented here are based on first-principles calculations employing the generalized gradient approximation (GGA), see Sec. II for more details.

substrate, while the scan at $150 \mathrm{~K}$ also shows an additional peak at $\sim 35^{\circ}$. Also shown are the calculated peak positions and relative intensities of three lowest-energy collinear spin configurations found from DFT calculations [see Fig. 3(b)]. Note that AFM $[0001]_{4}^{A}$ is the only configuration out of these three showing an extra peak due to the spin dependence of the Mn-Ga-Mn distances, which is within $1.2^{\circ}$ of the measured extra peak. Furthermore, the inset zooms in on the position of the 0006 peak showing that the lattice contracts by $\sim 0.3 \%$ from RT in the $c$ direction. Figure 4(b) shows the remanence measured with MOKE as a function of temperature along with the relative change in the out-of-plane lattice parameter deduced from the position of the 0006 peak. MOKE clearly confirms that below $\sim 250 \mathrm{~K}$ the magnetism has an FM component. This FM component can arise due to the canting of spins from their ideal positions in $\mathrm{AFM}[0001]_{4}^{A}$ configuration, as will be elucidated below. As the temperature increases, the material undergoes a sharp magnetic transition [not following the $\left(T_{\mathrm{c}}-T\right)^{\beta}$ dependence characteristic of FMs], which is accompanied by an expansion in the out-of-plane direction.

The additional peak observed by XRD at $35.1^{\circ}$ in Fig. 4(a) along with the change in lattice parameter $c$ corroborates the findings from calculations where we expect the lattice to change out-of-plane, beyond the effect of thermal expansion, when moving between spin configurations, and also to display different symmetry depending on the configuration. The thermal expansion of the substrate is small in this temperature range $\left(0.5-10 \cdot 10^{-6} \mathrm{~K}^{-1}\right)$ and any contraction of the substrate with decreasing temperature will expand the film out-of-plane, assuming that the film is "fixed" to the substrate, which is opposite to our observations. The anisotropic change of the crystal structure seems to be a direct consequence of the magnetic configuration, contrary to commonly observed changes in magnetic behavior following a change in structure, and could therefore be characterized as magnetically driven. Similar behavior, yet isostructural, has been observed in the closely related $\mathrm{Mn}_{3} \mathrm{GaC}$ which contracts by $0.5 \%$ upon increasing temperature when it undergoes a first-order AFM to FM transition at $160 \mathrm{~K}$ [26].

It should be noted that, except for FM, none of the collinear configurations considered display any net magnetization in the absence of field. The measured magnetic moment of $\sim 1.7 \mu_{B}$ per $\mathrm{Mn}$ at $3 \mathrm{~K}$ and $5 \mathrm{~T}$ does come close to the predicted moment of 1.83 (GGA) and 1.59 (LDA) $\mu_{B}$ per Mn in FM. For details see Table S1 [55]. However, this is almost an order of magnitude larger than the measured remanent moment $m_{r}$ of $\sim 0.3 \mu_{B}$ per Mn and thus a more detailed look at the spin configuration is required to explain the magnetic behavior.

\section{Theoretical ground-state search}

Since all the identified low-energy collinear spin configurations have parallel spin directions within a Mn-C-Mn trilayer, i.e., strong intralayer FM coupling, we simplify the picture by representing the local $\mathrm{Mn}$ moments within a trilayer as a supermoment. This is additionally strengthened by the small 


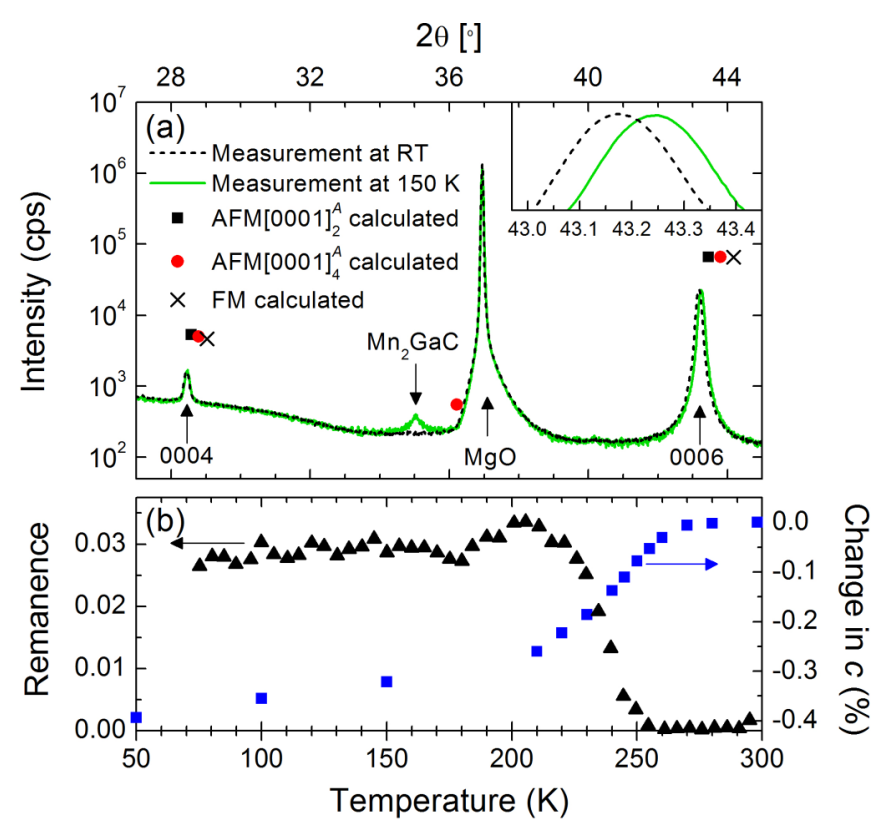

FIG. 4. (a) $2 \theta-\omega$ scans of $\mathrm{Mn}_{2} \mathrm{GaC}$ at room temperature (RT) and $150 \mathrm{~K}$. A shift in the 0006 peak position is accompanied by a new peak appearing at $\sim 35^{\circ}$ for the $150 \mathrm{~K}$ scan, compared to RT. The theoretical peak positions and their relative intensities for three different spin configurations are also presented. The inset shows a close-up of the 0006 peak shift. (b) Remanent moment $m_{r}$ measured with MOKE (in arbitrary units) as a function of temperature $T$, and the relative change in $c$-lattice parameter with respect to measurement at RT.

interlayer distance $d_{X}$ of $2.09 \AA$, which corresponds to a Mn-Mn distance of $2.67 \AA$, in line with results from Gercsi et al. [29] that show an interatomic distance of $2.88 \AA$ to be the threshold for FM favored coupling in Mn-based materials. Hence, we performed a magnetic ground-state search based on this subset of magnetic structures using a Heisenberg Monte Carlo approach together with the simplified supermoment representation; thus focusing on interactions across the $A$ layer. The aim is to find additional, possibly more complex, magnetic ground-state configurations using a Heisenberg Hamiltonian represented by a chain of supermoments.

Figure 5(a) shows the total net moment as a function of volume where a value of 1 corresponds to FM and 0 to AFM (collinear and noncollinear) based on MEI extracted from GGA and the local spin density approximation (LSDA) calculations (see Sec. II for details). Note that the volume change can mainly be attributed to the lattice parameter $c$. Figure 5(b) illustrates six selected low-energy configurations based on the Heisenberg Monte Carlo simulations at different volumes, increasing from configuration I to configuration VI. Focusing on GGA results, at volumes $\leqslant 43.0 \AA^{3} / \mathrm{fu}$ all supermoments are parallel with a net magnetization of 1 , illustrated by state I in Fig. 5(b). From 43.1 to 43.3 there is a transition from an FM state, via a transition state III in the form of a spin wave, to configurations with zero, or close to zero, net magnetization, illustrated by the spin spirals IV-VI. Corresponding behavior is found for LSDA but shifted to larger volumes. In general LSDA gives a better approximation for
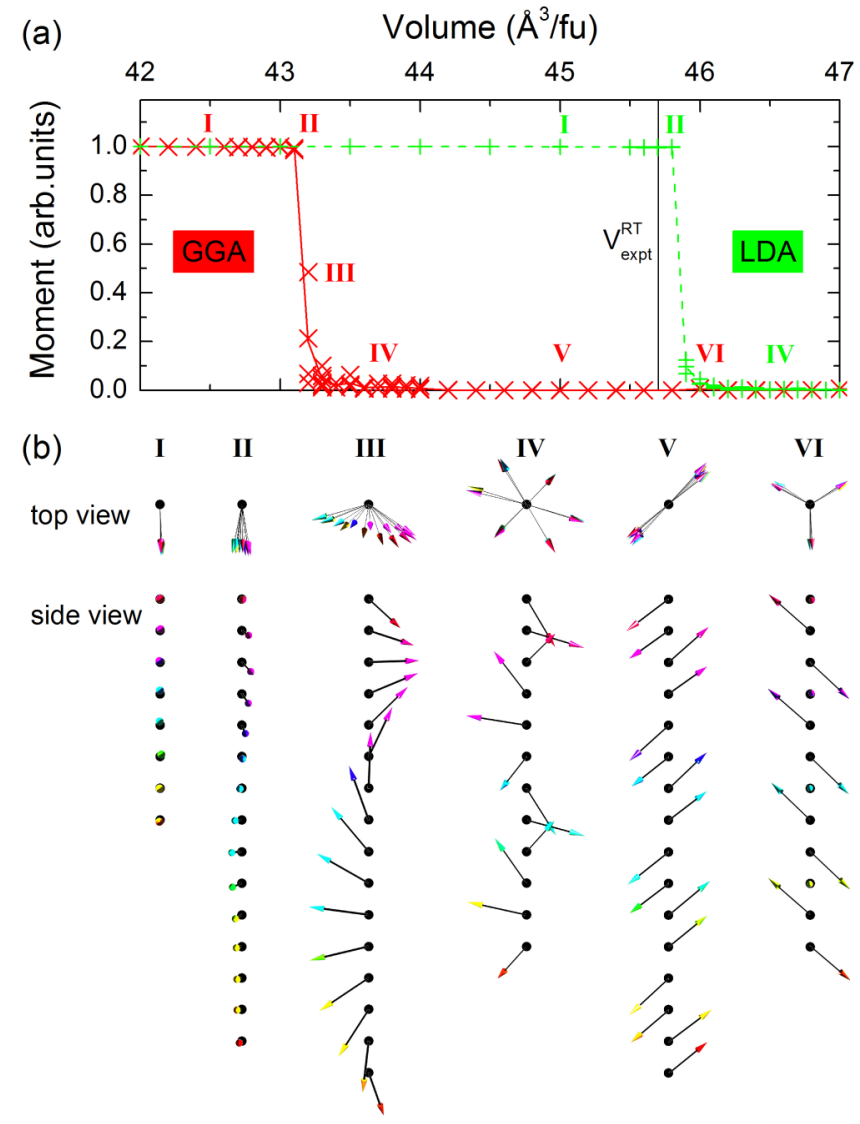

FIG. 5. Heisenberg Monte Carlo simulations with (a) net moment of lowest-energy configurations as a function of volume. Results from different chain lengths for GGA $(\times)$ and LDA $(+)$ based MEI, with corresponding average in solid red and dashed green lines, respectively. (b) Selected lowest-energy spin spirals at different volumes, as indicated in (a), where each arrow represents the spin direction of a Mn-C-Mn supermoment.

magnetic properties while GGA is more reliable for structure relaxation [64]. Noticeable from Fig. 5 is the sensitivity of the magnetic configuration to the volume, i.e., a slight change of volume, for instance due to thermal expansion, can alter the magnetic behavior significantly. It also indicates that by inducing a magnetic transition with field the volume can be influenced, expressed solely as a change in the out-of-plane lattice parameter $c$ as seen in Figs. 3(d) and 4(b).

\section{E. Canted AFM spin structures}

A small set of representative spin spiral configurations was selected for evaluation with first-principles calculations. Of special interest is spin spiral $\mathrm{V}$, which resembles AFM $[0001]_{4}^{A}$ though with supermoments slightly canted by $\pm 7^{\circ}$. Furthermore, to account for the measured finite net moment, which none of spin spirals IV to VI possess, and inspired by spin spiral $\mathrm{V}$, we calculated a series of in-plane spin canting angles $\theta_{\mathrm{c}}$, from $0^{\circ}\left(\mathrm{AFM}[0001]_{4}^{A}\right)$ to $90^{\circ}(\mathrm{FM})$. This transition is illustrated in Fig. 6(a), where the relation between the supermoment picture and the crystal configuration is given for the AFM $[0001]_{4}^{A}$ and FM spin configuration. In Fig. 6(b) the energy relative to $\mathrm{AFM}[0001]_{4}^{A}$ is given as a function of canting 
(a)
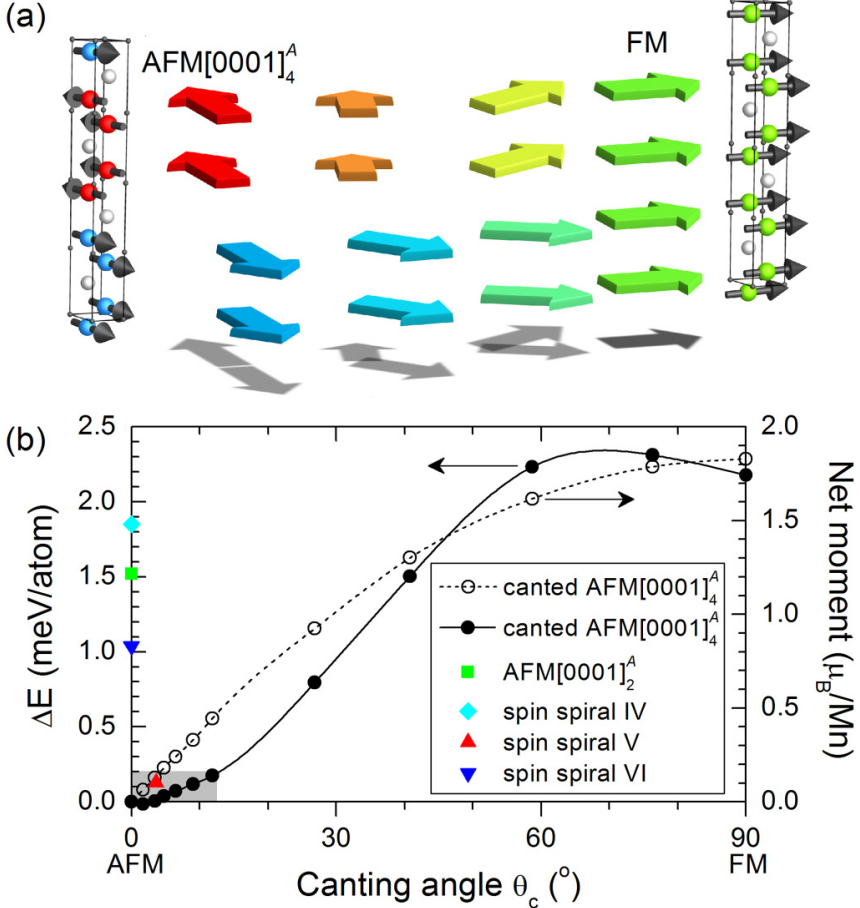

FIG. 6. Schematic of a possible transition between magnetic states and explanation for the observed FM response. (a) Schematic of a canting transition from AFM $[0001]_{4}^{A}$ to FM using the coarse-grained model with $\mathrm{Mn}$ moments in a Mn-C-Mn trilayer represented by a supermoment. The crystal configuration of AFM $[0001]_{4}^{A}$ and FM is also seen as a reference. (b) Change in energy of the canted AFM $[0001]_{4}^{A}$ with canting angle (filled circles) along with the corresponding net moment (open circles). Note that $\theta_{c}=90$ corresponds to an FM state. The gray box indicates the region where the angle is degenerate to within $0.2 \mathrm{meV} /$ atom. Also shown are the energies for selected low-energy spin spirals IV $(\diamond), \mathrm{V}(\boldsymbol{\Delta})$, and VI $(\boldsymbol{\nabla})$ as well as for AFM[0001 $]_{4}^{A}(\square)$, relative to that of AFM[0001 $]_{4}^{A}$.

angle as well as the corresponding calculated net moments. Also, the energies for selected configurations from Fig. 5(b) are displayed, for comparison. For details of noncollinear spin structures see Table S2 [55].

For $\theta_{\mathrm{c}}<12^{\circ}$ the energies are considered nearly degenerate, within $0.2 \mathrm{meV} /$ atom, possibly with a presence of a minimum at nonzero canting angle, which indicates the energetical preference or minimal cost in energy needed to cant the spins away from the collinear AFM, state, resulting in a measurable remanent moment. This could explain the measured FM component below $250 \mathrm{~K}$. The subsequent rise in magnetization would then result in further canting of the spins, beyond the degenerate angle $\theta_{\mathrm{c}}<12^{\circ}$, at a higher cost in energy, i.e., applied magnetic field. We can then speculate that as the temperature increases the volume expands, thus moving the system further away from the FM regime as seen in Fig. 5(a). The energy needed to cant the spins beyond $12^{\circ}$ therefore increases, resulting in the observed magnetization behavior at $5 \mathrm{~T}$, but the degenerate angle remains constant. This is supported by the nearly constant $m_{r}$ versus $\mathrm{T}$ behavior seen in the MOKE measurements in Fig. 4(b).

Looking at the magnetic behavior at and above $230 \mathrm{~K}$, the measurements show both a change in structure as well as a large change in magnetic behavior. This is seen in Fig. 4 by the disappearance of the extra peak, out-of-plane expansion, and the disappearance of the remanence. This indicates that the spin configuration has changed to an AFM state of higher symmetry without influence of a magnetic field due to volume expansion with increased temperature. Above $250 \mathrm{~K}$, as a field is applied the magnetization increases slightly until there is a transition. This can be understood in terms of a metamagnetic transition from a noncanted to canted AFM state [18]. Figure 6(b) also shows energies of selected spin spirals, all close in energy to the collinear configurations. This illustrates that a large amount of different spin configurations may be of relevance for the ground state of $\mathrm{Mn}_{2} \mathrm{GaC}$.

The suggested canted AFM[0001 $]_{4}^{A}$ configuration is supported by its calculated low-energy and the extra diffraction peak measured. The fact that the extra peak is significantly shifted with respect to the calculated position actually indicates that the correct magnetic configurations are not among those considered. However, this additional peak is consistent with other configurations with similar attributes as AFM[0001 $]_{4}^{A}$, i.e., with at least two consecutive trilayers of the same or close to the same spin orientation. This would promote changes in the crystal structure, and with degenerate angles that would give a net moment upon a small perturbation, such as various complex spin spirals. The magnetic behavior at $3 \mathrm{~K}$ further supports a canted AFM state, as the net moment at $5 \mathrm{~T}$ comes close to the theoretical value of an FM state. We do not, however, have an FM state as the moments only becomes close to fully collinear under a high magnetic field. The same applies above $230 \mathrm{~K}$ where we cannot distinguish between spin configurations with a retained MAX phase structure, i.e., at most one consecutive trilayer of the same spin orientation (e.g., AFM[0001 $]_{2}^{A}$, spin spiral IV, VI). However, we do know that the material retains spin order up to RT, i.e., it is not PM.

\section{DISCUSSION}

The presented results show that $\mathrm{Mn}_{2} \mathrm{GaC}$ exhibits many of the characteristics which are typical of magnetocaloric [24,29] and magnetoelectric [16,18,20,36] materials. First, it displays a rich magnetic phase diagram allowing for many different types of spin states, close in energy, some degenerate. The transitions between these states can occur by altering temperature due their volume dependence or by applied magnetic, or possibly electric, field due to their degeneracy. Second, accompanied by such transitions are drastic anisotropic changes in the crystal structure of the material, both in terms of contraction/expansion as well as symmetry changes. The $0.2 \%$ change in the out-of-plane $c$ axis at the transition between 230 and $250 \mathrm{~K}$ is a considerably large effect compared to what is commonly observed for systems that display magnetostriction. On top of this temperature-driven volume change, the asymmetric change of the crystal structure below $230 \mathrm{~K}$, with the appearance of a diffraction peak at $35.1^{\circ}$, is expected to be solely a consequence of spin ordering between Mn-C-Mn trilayers, contrary to commonly observed changes in magnetic ordering due to structural changes $[18,29]$. These drastic changes indicate that the magnetic state of the material could also be influenced by an applied pressure or through the introduction of stress. By compressing 
the lattice in the $c$ direction the Mn-Mn interlayer distance is reduced and thereby strengthening FM coupling across the $A$ layer [31] thus shifting the occurrence of magnetic transitions. This could be realized by the introduction of in-plane tensile strain, e.g., from bending the material or by deposition on a substrate with a larger in-plane lattice parameter.

The ability to alloy on the $M, A$, or $X$ site [42,65-71] or create artificial superstructures composed of different MAX phases would allow for possible routes to affect/tune electronic, magnetic, and mechanical properties without affecting the inherent atomically laminated structure. In addition, a new family of two-dimensional (2D) materials known as MXenes was recently discovered, realized by etching the $A$ layers from the MAX phase [72,73]. The capability of making MXenes from magnetic MAX phases, in combination with the strong intralayer ferromagnetic coupling within Mn-C-Mn trilayers of $\mathrm{Mn}_{2} \mathrm{GaC}$, which persists above room temperature opens the possibility to produce 2D ferromagnetic crystals [72,74], to date still missing due to the chemical instability of such compounds. Mapping out the rich magnetic phase diagram of $\mathrm{Mn}_{2} \mathrm{GaC}$ offers possibilities to fully explain the magnetism for this family of atomically laminated materials as well as deepening the understanding of layered magnetic systems in general. In parallel, transport properties and possible caloric effect should be studied to establish whether these materials could be used for future magnetoelectric or magnetocaloric applications.

\section{ACKNOWLEDGMENTS}

The research leading to these results has received funding from the European Research Council under the European Communities Seventh Framework Programme (FP7/20072013)/ERC Grant No. 258509. J. R. acknowledges funding from the Swedish Research Council (VR) Grant No. 6422013-8020, from the KAW Fellowship program, and from the SSF synergy grant FUNCASE. B. A. is grateful for funding from the Swedish Research Council (VR) Grant No. 621-20114417. I. A. A. acknowledges the support from VR Grant No. 621-2011-4426, the Grant of the Russian Federation Ministry for Science and Education (Grant No. 14.Y26.31.0005) and The Tomsk State University Academic D. I. Mendeleev Fund Program. Calculations were performed utilizing supercomputer resources supplied by the Swedish National Infrastructure for Computing (SNIC) at the National Supercomputer Centre (NSC), the High Performance Computing Center North (HPC2N).

M.D. and A.S.I. contributed equally to this work.
[1] E. Ising, Z. Physik 31, 253 (1925).

[2] N. D. Mermin and H. Wagner, Phys. Rev. Lett. 17, 1133 (1966).

[3] M. Weinert and A. J. Freeman, J. Magn. Magn. Mater. 38, 23 (1983).

[4] J. Dorantes-Dávila and G. M. Pastor, Phys. Rev. Lett. 81, 208 (1998).

[5] P. Kurz, G. Bihlmayer, and S. Blugel, J. Appl. Phys. 87, 6101 (2000).

[6] L. J. De Jongh and A. R. Miedema, Adv. Phys. 23, 1 (1974).

[7] F. J. Himpsel, J. E. Ortega, G. J. Mankey, and R. F. Willis, Adv. Phys. 47, 511 (1998).

[8] M. Pratzer, H. J. Elmers, M. Bode, O. Pietzsch, A. Kubetzka, and R. Wiesendanger, Phys. Rev. Lett. 87, 1272011 (2001).

[9] M. Wasniowska, S. Schröder, P. Ferriani, and S. Heinze, Phys. Rev. B 82, 0124021 (2010).

[10] A. S. Arrott, B. Heinrich, S. T. Purcell, J. F. Cochran, and K. B. Urquhart, J. Appl. Phys. 61, 3721 (1987).

[11] W. L. O'Brien and B. P. Tonner, J. Vac. Sci. Technol. A 13, 1544 (1995).

[12] X. Z. Yu, Y. Onose, N. Kanazawa, J. H. Park, J. H. Han, Y. Matsui, N. Nagaosa, and Y. Tokura, Nature (London) 465, 901 (2010).

[13] X. Fabrèges, S. Petit, I. Mirebeau, S. Pailhès, L. Pinsard, A. Forget, M. T. Fernandez-Diaz, and F. Porcher, Phys. Rev. Lett. 103, 0672041 (2009).

[14] I. Mirebeau and S. Petit, J. Magn. Magn. Mater. 350, 209 (2014).

[15] W. Wulfhekel and C. L. Gao, J. Phys. Cond. Matter 22, 0840211 (2010).

[16] Y. Tokura and S. Seki, Adv. Mater. 22, 1554 (2010).

[17] J. Dho, W. S. Kim, and N. H. Hur, Phys. Rev. Lett. 89, 0272021 (2002).
[18] S.-J. Ahn, J.-H. Lee, Y. K. Jeong, E.-H. Na, Y. M. Koo, and H. M. Jang, Mater. Chem. Phys. 138, 929 (2013).

[19] S.-W. Cheong and M. Mostovoy, Nat. Mater. 6, 13 (2007).

[20] R. Ramesh and N. A. Spaldin, Nat. Mater. 6, 21 (2007).

[21] P. Grünberg, R. Schreiber, Y. Pang, M. B. Brodsky, and H. Sowers, Phys. Rev. Lett. 57, 2442 (1986).

[22] G. Binasch, P. Grünberg, F. Saurenbach, and W. Zinn, Phys. Rev. B 39, 4828 (1989).

[23] M.-H. Phan and S.-C. Yu, J. Magn. Magn. Mater. 308, 325 (2007).

[24] X. Moya, S. Kar-Narayan, and N. D. Mathur, Nat. Mater. 13, 439 (2014).

[25] T. Tohei, H. Wada, and T. Kanomata, J. Appl. Phys. 94, 1800 (2003).

[26] Ö. Çakır, M. Acet, M. Farle, and A. Senyshyn, J. Appl. Phys. 115, 0439131 (2014).

[27] O. Tegus, E. Bruck, K. H. J. Buschow, and F. R. de Boer, Nature (London) 415, 150 (2002).

[28] H. Wada and Y. Tanabe, Appl. Phys. Lett. 79, 3302 (2001).

[29] Z. Gercsi and K. G. Sandeman, Phys. Rev. B 81, 224426 (2010).

[30] J. B. Staunton, M. dos Santos Dias, J. Peace, Z. Gercsi, and K. G. Sandeman, Phys. Rev. B 87, 0604041 (2013).

[31] Z. Gercsi, K. Hono, and K. G. Sandeman, Phys. Rev. B 83, 1744031 (2011).

[32] W. Kuch, L. I. Chelaru, F. Offi, J. Wang, M. Kotsugi, and J. Kirschner, Nat. Mater. 5, 128 (2006).

[33] M. N. Baibich, J. M. Broto, A. Fert, F. Nguyen Van Dau, F. Petroff, P. Etienne, G. Creuzet, A. Friederich, and J. Chazelas, Phys. Rev. Lett. 61, 2472 (1988).

[34] C. Quirós, J. I. Martín, L. Zárate, M. Vélez, and J. M. Alameda, Phys. Rev. B 71, 0244231 (2005). 
[35] M. Fiebig, D. Fröhlich, K. Kohn, S. Leute, T. Lottermoser, V. V. Pavlov, and R. V. Pisarev, Phys. Rev. Lett. 84, 5620 (2000).

[36] T. Kimura, T. Goto, H. Shintani, K. Ishizaka, T. Arima, and Y. Tokura, Nature (London) 426, 55 (2003).

[37] A. S. Ingason, A. Petruhins, M. Dahlqvist, F. Magnus, A. Mockute, B. Alling, L. Hultman, I. A. Abrikosov, P. O. Å. Persson, and J. Rosen, Mater. Res. Lett. 2, 89 (2014).

[38] M. W. Barsoum and M. Radovic, Annu. Rev. Mater. Res. 41, 195 (2011).

[39] M. W. Barsoum, Prog. Solid State Chem. 28, 201 (2000).

[40] P. Eklund, M. Beckers, U. Jansson, H. Högberg, and L. Hultman, Thin Solid Films 518, 1851 (2010).

[41] M. Dahlqvist, B. Alling, I. A. Abrikosov, and J. Rosen, Phys. Rev. B 84, 220403 (2011).

[42] A. S. Ingason, A. Mockute, M. Dahlqvist, F. Magnus, S. Olafsson, U. B. Arnalds, B. Alling, I. A. Abrikosov, B. Hjörvarsson, P. O. A. Persson, and J. Rosen, Phys. Rev. Lett. 110, 195502 (2013).

[43] S. Lin, P. Tong, B. S. Wang, Y. N. Huang, W. J. Lu, D. F. Shao, B. C. Zhao, W. H. Song, and Y. P. Sun, J. Appl. Phys. 113, 053502 (2013).

[44] Z. Liu, T. Waki, Y. Tabata, and H. Nakamura, Phys. Rev. B 89, 054435 (2014).

[45] A. Mockute, J. Lu, E. J. Moon, M. Yan, B. Anasori, S. J. May, M. W. Barsoum, and J. Rosen, Mater. Res. Lett. 3, 16 (2014).

[46] A. Mockute, P. O. Å. Persson, F. Magnus, A. S. Ingason, S. Olafsson, L. Hultman, and J. Rosen, Phys. Status Solidi Rapid Res. Lett. 8, 420 (2014).

[47] Q. Z. Tao, C. F. Hu, S. Lin, H. B. Zhang, F. Z. Li, D. Qu, M. L. Wu, Y. P. Sun, Y. Sakka, and M. W. Barsoum, Mater. Res. Lett. 2, 192 (2014).

[48] M. Jaouen, M. Bugnet, N. Jaouen, P. Ohresser, V. Mauchamp, T. Cabioc'h, and A. Rogalev, J. Phys. Cond. Matter 26, 176002 (2014).

[49] M. Jaouen, P. Chartier, T. Cabioc'h, V. Mauchamp, G. André, and M. Viret, J. Am. Ceram. Soc. 96, 3872 (2013).

[50] Z. Liu, T. Waki, Y. Tabata, K. Yuge, H. Nakamura, and I. Watanabe, Phys. Rev. B 88, 134401 (2013).

[51] B. L. Gyorffy, A. J. Pindor, J. Staunton, G. M. Stocks, and H. Winter, J. Phys. F: Met. Phys. 15, 1337 (1985).

[52] B. Alling, T. Marten, and I. A. Abrikosov, Phys. Rev. B 82, 184430 (2010).
[53] A. Zunger, S. H. Wei, L. G. Ferreira, and J. E. Bernard, Phys. Rev. Lett. 65, 353 (1990).

[54] B. Alling, A. V. Ruban, A. Karimi, O. E. Peil, S. I. Simak, L. Hultman, and I. A. Abrikosov, Phys. Rev. B 75, 0451231 (2007).

[55] See Supplemental Material at http://link.aps.org/supplemental/ 10.1103/PhysRevB.93.014410 for further experimental and theoretical details.

[56] P. E. Blöchl, Phys. Rev. B 50, 17953 (1994).

[57] G. Kresse and J. Hafner, Phys. Rev. B 48, 13115 (1993).

[58] G. Kresse and D. Joubert, Phys. Rev. B 59, 1758 (1999).

[59] J. P. Perdew, K. Burke, and M. Ernzerhof, Phys. Rev. Lett. 77, 3865 (1996).

[60] H. J. Monkhorst and J. D. Pack, Phys. Rev. B 13, 5188 (1976).

[61] S. Mankovsky, S. Polesya, S. Bornemann, J. Minár, F. Hoffmann, C. H. Back, and H. Ebert, Phys. Rev. B 84, 201201 (2011).

[62] J. W. D. Connolly and A. R. Williams, Phys. Rev. B 27, 5169 (1983).

[63] A. Lindmaa, R. Lizárraga, E. Holmström, I. A. Abrikosov, and B. Alling, Phys. Rev. B 88, 054414 (2013).

[64] A. V. Ruban, S. Shallcross, S. I. Simak, and H. L. Skriver, Phys. Rev. B 70, 1251151 (2004).

[65] A. Petruhins, A. S. Ingason, J. Lu, F. Magnus, S. Olafsson, and J. Rosen, J. Mater. Sci. 50, 4495 (2015).

[66] R. Meshkian, A. S. Ingason, U. B. Arnalds, F. Magnus, J. Lu, and J. Rosen, APL Materials 3, 076102 (2015).

[67] Z. Liu et al., Acta Mater. 73, 186 (2014).

[68] E. N. Caspi, P. Chartier, F. Porcher, F. Damay, and T. Cabioc'h, Mater. Res. Lett. 3, 100 (2015).

[69] B. Anasori et al., J. Appl. Phys. 118, 094304 (2015).

[70] T. Cabioch, P. Eklund, V. Mauchamp, M. Jaouen, and M. W. Barsoum, J. Eur. Ceram. Soc. 33, 897 (2013).

[71] T. Scabarozi, A. Ganguly, J. D. Hettinger, S. E. Lofland, S. Amini, P. Finkel, T. El-Raghy, and M. W. Barsoum, J. Appl. Phys. 104, 073713 (2008).

[72] M. Naguib, M. Kurtoglu, V. Presser, J. Lu, J. Niu, M. Heon, L. Hultman, Y. Gogotsi, and M. W. Barsoum, Adv. Mater. 23, 4248 (2011).

[73] B. Anasori, Y. Xie, M. Beidaghi, J. Lu, B. C. Hosler, L. Hultman, P. R. C. Kent, Y. Gogotsi, and M. W. Barsoum, ACS Nano 9, 9507 (2015).

[74] B. Sachs, T. O. Wehling, K. S. Novoselov, A. I. Lichtenstein, and M. I. Katsnelson, Phys. Rev. B 88, 201402 (2013). 